\title{
Research Article \\ Dynamics of a Predator-Prey System Concerning Biological and Chemical Controls
}

\author{
Hye Kyung Kim ${ }^{1}$ and Hunki Baek ${ }^{2}$ \\ ${ }^{1}$ Department of Mathematics Education, Catholic University of Daegu, Kyongsan \\ 712-702, Republic of Korea \\ ${ }^{2}$ Department of Mathematics, Kyungpook National University, Daegu 702-701, Republic of Korea
}

Correspondence should be addressed to Hunki Baek, hkbaek@knu.ac.kr

Received 25 August 2010; Accepted 13 November 2010

Academic Editor: Mohamed A. El-Gebeily

Copyright (C) 2010 H. K. Kim and H. Baek. This is an open access article distributed under the Creative Commons Attribution License, which permits unrestricted use, distribution, and reproduction in any medium, provided the original work is properly cited.

\begin{abstract}
We investigate an impulsive predator-prey system with Monod-Haldane type functional response and control strategies, especially, biological and chemical controls. Conditions for the stability of the prey-free positive periodic solution and for the permanence of the system are established via the Floquet theory and comparison theorem. Numerical examples are also illustrated to substantiate mathematical results and to show that the system could give birth to various kinds of dynamical behaviors including periodic doubling, and chaotic attractor. Finally, in discussion section, we consider the dynamic behaviors of the system when the growth rate of the prey varies according to seasonal effects.
\end{abstract}

\section{Introduction}

In recent years controlling insects and other arthropods has become an increasingly complex issue. There are many ways that can be used to help control the population of insect pests. Integrated Pest Management (IPM) is a pest control strategy that uses an array of complementary methods: natural predators and parasites, pest-resistant varieties, cultural practices, biological controls, various physical techniques, and the strategic use of pesticides.

Chemical control is one of simple methods for pest control. Pesticides are often useful because they quickly kill a significant portion of a pest population. However, there are many deleterious effects associated with the use of chemicals that need to be reduced or eliminated. These include human illness associated with pesticide applications, insect resistance to insecticides, contamination of soil and water, and diminution of biodiversity. As a result, it is required that we should combine pesticide efficacy tests with other ways of control. Another important way to control pest populations is biological control. It is defined as the reduction 


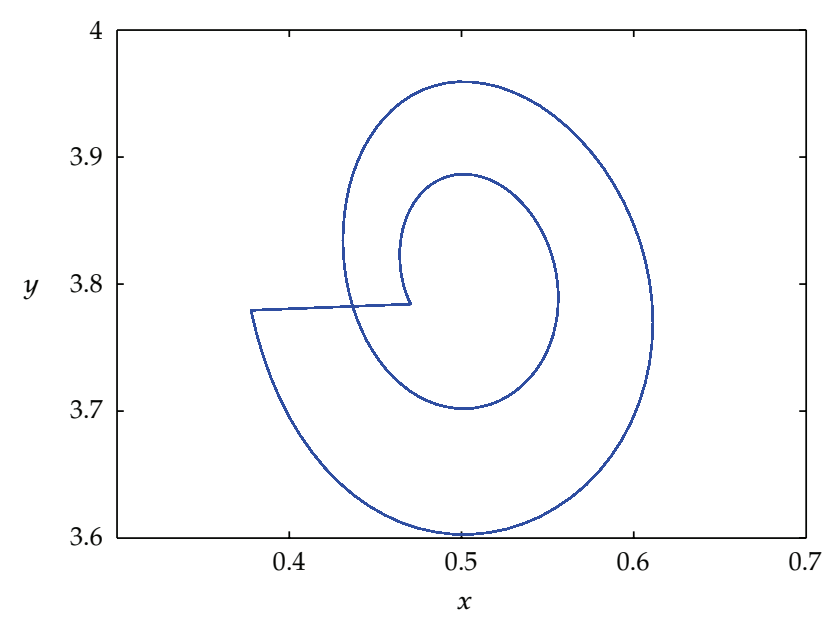

Figure 1: Phase portrait of a $T$-period solution of (1.3) for $q=0$.

of pest populations by natural enemies and typically involves an active human role. Natural enemies of insect pests, also known as biological control agents, include predators, parasites, and pathogens. Virtually all pests have some natural enemies, and the key to successful pest control is to identify the pest and its natural enemies and release them at fixed times for pest control. Biological control can be an important component of Integrated Pest Management (IPM) programs. Such different pest control tactics should work together rather than against each other to accomplish an IPM program successfully $[1,2]$.

On the other hand, the relationship between pest and natural enemy can be expressed a predator(natural enemy)-prey(pest) system mathematically as follows:

$$
\begin{gathered}
x^{\prime}(t)=a x(t)\left(1-\frac{x(t)}{K}\right)-y P(x, y), \\
y^{\prime}(t)=-d y(t)+e y P(x, y), \\
x(0)=x_{0} \geq 0, \quad y(0)=y_{0}>0,
\end{gathered}
$$

where $x(t)$ and $y(t)$ represent the population density of the prey and the predator at time $t$, respectively. Usually, $K$ is called the carrying capacity of the prey. The constant $a$ is called intrinsic growth rate of the prey. The constants $e, d$ are the conversion rate and the death rate of the predator, respectively. The function $P$ is the functional response of the predator which means prey eaten per predator per unit of time. Many scholars have studied such predatorprey systems with functional response, such as Holling-type [3-5], Beddington-type [6-9], and Ivlev-type [10-12]. One of well-known function response is of Monod-Haldane type [4, 5, 13]. The predator-prey system with Monod-Haldane type is described by the following 


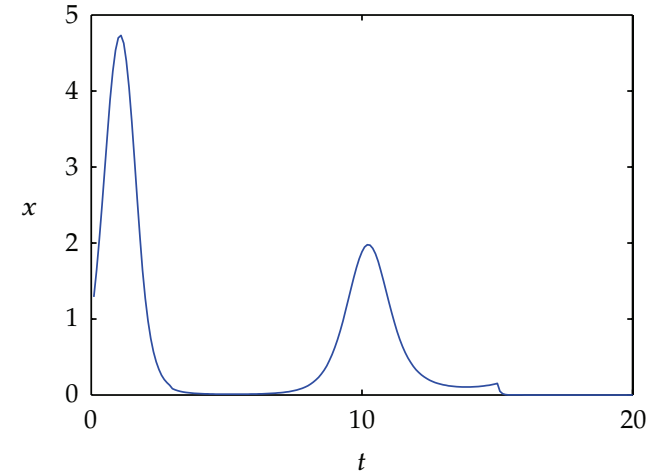

(a)

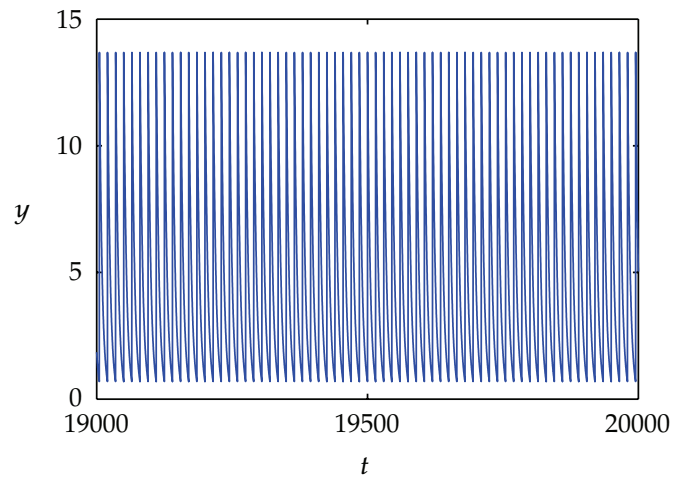

(b)

Figure 2: Dynamical behavior of (1.3) with $q=13$. (a) $x$ is plotted. (b) $y$ is plotted.

differential equation:

$$
\begin{aligned}
& x^{\prime}(t)=a x(t)\left(1-\frac{x(t)}{K}\right)-\frac{c x(t) y(t)}{1+b x^{2}(t)} \\
& y^{\prime}(t)=-d y(t)+\frac{e x(t) y(t)}{1+b x^{2}(t)} \\
& \left(x\left(0^{+}\right), y\left(0^{+}\right)\right)=\left(x_{0}, y_{0}\right)=\mathbf{x}_{0} .
\end{aligned}
$$

Therefore, to accomplish the aims discussed above, we need to consider impulsive differential equation as follows:

$$
\begin{aligned}
& x^{\prime}(t)=a x(t)\left(1-\frac{x(t)}{K}\right)-\frac{c x(t) y(t)}{1+b x^{2}(t)}, \quad t \neq n T, \quad t \neq(n+\tau-1) T, \\
& y^{\prime}(t)=-d y(t)+\frac{e x(t) y(t)}{1+b x^{2}(t)} \\
& x\left(t^{+}\right)=\left(1-p_{1}\right) x(t), \quad t=(n+\tau-1) T, \\
& y\left(t^{+}\right)=\left(1-p_{2}\right) y(t), \\
& x\left(t^{+}\right)=x(t), \\
& y\left(t^{+}\right)=y(t)+q, \quad t=n T, \\
& \left(x\left(0^{+}\right), y\left(0^{+}\right)\right)=\left(x_{0}, y_{0}\right)=x_{0},
\end{aligned}
$$

where the parameters $0 \leq \tau<1$ and $T>0$ are the periods of the impulsive immigration or stock of the predator, $0 \leq p_{1}, p_{2}<1$ present the fraction of the prey which dies due to the harvesting or pesticides and so forth, and $q$ is the size of immigration or stock of the predator.

In fact, impulsive control methods can be found in almost every field of applied sciences. The theoretical investigation and its application analysis can be found in Bainov and 


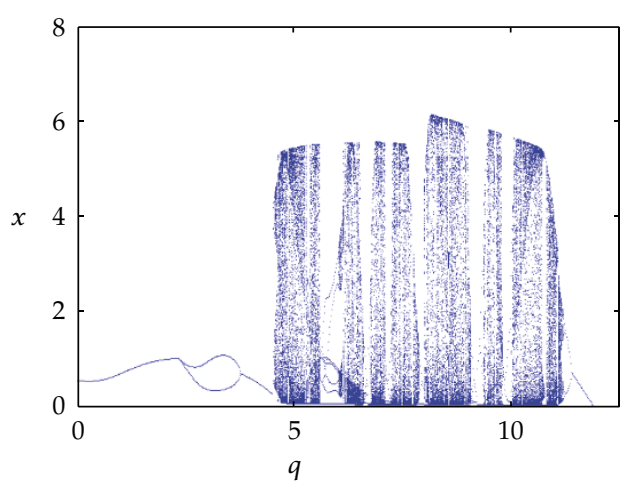

(a)

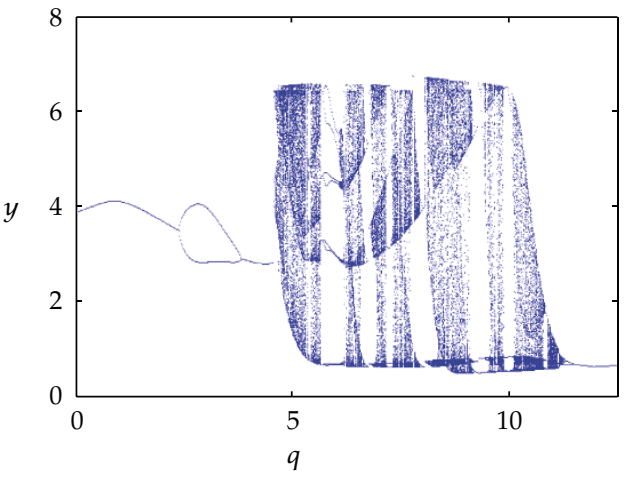

(b)

Figure 3: Bifurcation diagrams of (1.3) for $q$ ranging from $0<q<13$. (a) $x$ is plotted. (b) $y$ is plotted.

Simeonov [14], Lakshmikantham et al. [15]. Moreover, the impulsive differential equations dealing with biological population dynamics are literate in [16-21]. In particular, Zhang et al. [20] studied the system (1.3) without chemical control. That is, $p_{1}=p_{2}=0$. They investigated the abundance of complex dynamics of the system (1.3) theoretically and numerically.

The main purpose of this paper is to investigate the dynamics of the system (1.3). In Section 3, we study qualitative properties of the system (1.3). In fact, we show the local stability of the prey-free periodic solution under some conditions and give a sufficient condition for the permanence of the system (1.3) by applying the Floquet theory. In Section 4 we numerically investigate the system (1.3) to figure out the influences of impulsive perturbations on inherent oscillation. Finally, in Section 5, we consider the dynamic behaviors of the system when the growth rate of the prey varies according to seasonal effects.

\section{Basic Definitions and Lemmas}

Before stating our main results, firstly, we give some notations, definitions and lemmas which will be useful for our main results.

Let $\mathbb{R}_{+}=[0, \infty), \mathbb{R}_{+}^{*}=(0, \infty)$ and $\mathbb{R}_{+}^{2}=\left\{\mathbf{x}=(x(t), y(t)) \in \mathbb{R}^{2}: x(t), y(t) \geq 0\right\}$. Denote $\mathbb{N}$ as the set of all of nonnegative integers and $f=\left(f_{1}, f_{2}\right)^{T}$ as the right hand of the system (1.3). Let $V: \mathbb{R}_{+} \times \mathbb{R}_{+}^{2} \rightarrow \mathbb{R}_{+}$, then $V$ is said to be in a class $V_{0}$ if

(1) $V$ is continuous in $((n-1) T,(n+\tau-1) T] \times \mathbb{R}_{+}^{2}$ and $((n+\tau-1) T, n T] \times \mathbb{R}_{+}^{2}$,

$$
\lim _{(t, \mathbf{y}) \rightarrow\left((n+\tau-1) T^{+}, \mathbf{x}\right)} V(t, \mathbf{y}) V\left((n+\tau-1) T^{+}, \mathbf{x}\right)
$$

and $\lim _{(t, y) \rightarrow\left(n T^{+}, x\right)} V(t, \mathbf{x})=V\left(n T^{+}, \mathbf{y}\right)$ exists for each $\mathbf{x} \in \mathbb{R}_{+}^{2}$ and $n \in \mathbb{N}$;

(2) $V$ is locally Lipschitzian in $\mathbf{x}$. 
Definition 2.1. Let $V \in V_{0},(t, \mathbf{x}) \in((n-1) T,(n+\tau-1) T] \times \mathbb{R}_{+}^{2}$ and $((n+\tau-1) T, n T] \times \mathbb{R}_{+}^{2}$. The upper right derivative of $V(t, \mathbf{x})$ with respect to the impulsive differential system (1.3) is defined as

$$
D^{+} V(t, \mathbf{x})=\limsup _{h \rightarrow 0+} \frac{1}{h}[V(t+h, \mathbf{x}+h f(t, \mathbf{x}))-V(t, \mathbf{x})]
$$

It is from [15] that the smoothness properties of $f$ guarantee the global existence and uniqueness of solutions to the system (1.3).

We will use a comparison inequality of impulsive differential equations. Suppose that $g: \mathbb{R}_{+} \times \mathbb{R}_{+} \rightarrow \mathbb{R}$ satisfies the following hypotheses:

$(\mathrm{H}) \mathrm{g}$ is continuous on $((n-1) T,(n+\tau-1) T] \times \mathbb{R}_{+} \cup((n+\tau-1) T, n T] \times \mathbb{R}_{+}$and the limits $\lim _{(t, y) \rightarrow\left((n+\tau-1) T^{+}, x\right)} g(t, y)=g\left((n+\tau-1) T^{+}, x\right), \lim _{(t, y) \rightarrow\left(n T^{+}, x\right)} g(t, y)=g\left(n T^{+}, x\right)$ exist and are finite for $x \in \mathbb{R}_{+}$and $n \in \mathbb{N}$.

Lemma 2.2 (see [15]). Suppose that $V \in V_{0}$ and

$$
\begin{gathered}
D^{+} V(t, \mathbf{x}) \leq g(t, V(t, \mathbf{x})), \quad t \neq(n+\tau-1) T, \quad t \neq n T, \\
V\left(t, \mathbf{x}\left(t^{+}\right)\right) \leq \psi_{n}^{1}(V(t, \mathbf{x})), \quad t=(n+\tau-1) T, \\
V\left(t, \mathbf{x}\left(t^{+}\right)\right) \leq \psi_{n}^{2}(V(t, \mathbf{x})), \quad t=n T,
\end{gathered}
$$

where $g: \mathbb{R}_{+} \times \mathbb{R}_{+} \rightarrow \mathbb{R}$ satisfies $(H)$ and $\psi_{n}^{1}, \psi_{n}^{2}: \mathbb{R}_{+} \rightarrow \mathbb{R}_{+}$are nondecreasing for all $n \in \mathbb{N}$. Let $r(t)$ be the maximal solution for the impulsive Cauchy problem

$$
\begin{gathered}
u^{\prime}(t)=g(t, u(t)), \quad t \neq(n+\tau-1) T, \quad t \neq n T, \\
u\left(t^{+}\right)=\psi_{n}^{1}(u(t)), \quad t=(n+\tau-1) T, \\
u\left(t^{+}\right)=\psi_{n}^{2}(u(t)), \quad t=n T, \\
u\left(0^{+}\right)=u_{0} \geq 0,
\end{gathered}
$$

defined on $[0, \infty)$. Then $V\left(0^{+}, \mathbf{x}_{0}\right) \leq u_{0}$ implies that $V(t, \mathbf{x}(t)) \leq r(t), t \geq 0$, where $\mathbf{x}(t)$ is any solution of (2.3).

Similar result can be obtained when all conditions of the inequalities in the Lemma 2.2 are reversed. Note that if we have some smoothness conditions of $g(t, u(t))$ to guarantee the existence and uniqueness of the solutions for (2.4), then $r(t)$ is exactly the unique solution of (2.4).

From Lemma 2.2, it is easily proven that the following lemma holds.

Lemma 2.3. Let $\mathbf{x}(t)=(x(t), y(t))$ be a solution of the system (1.3). Then one has the following:

(1) if $\mathbf{x}\left(0^{+}\right) \geq 0$ then $\mathbf{x}(t) \geq 0$ for all $t \geq 0$;

(2) if $\mathbf{x}\left(0^{+}\right)>0$ then $\mathbf{x}(t)>0$ for all $t \geq 0$. 
It follows from Lemma 2.3 that the positive quadrant $\left(\mathbb{R}_{+}^{*}\right)^{2}$ is an invariant region of the system (1.3).

Even if the Floquet theory is well known, we would like to mention the theory to study the stability of the prey-free periodic solution as a solution of the system (1.3). For this, we present the Floquet theory for the linear $T$-periodic impulsive equation:

$$
\begin{gathered}
\frac{d x}{d t}=A(t) x(t), \quad t \neq \tau_{k}, t \in \mathbb{R}, \\
x\left(t^{+}\right)=x(t)+B_{k} x(t), \quad t=\tau_{k}, k \in \mathbb{Z} .
\end{gathered}
$$

Then we introduce the following conditions.

$\left(H_{1}\right) A(\cdot) \in \operatorname{PC}\left(\mathbb{R}, C^{n \times n}\right)$ and $A(t+T)=A(t)(t \in \mathbb{R})$, where $\operatorname{PC}\left(\mathbb{R}, C^{n \times n}\right)$ is a set of all piecewise continuous matrix functions which is left continuous at $t=\tau_{k}$, and $C^{n \times n}$ is a set of all $n \times n$ matrices.

$\left(H_{2}\right) B_{k} \in C^{n \times n}, \operatorname{det}\left(E+B_{k}\right) \neq 0, \tau_{k}<\tau_{k+1}(k \in \mathbb{Z})$.

$\left(H_{3}\right)$ There exists a $q \in \mathbb{N}$ such that $B_{k+q}=B_{k}, \tau_{k+q}=\tau_{k}+T(k \in \mathbb{Z})$.

Let $\Phi(t)$ be a fundamental matrix of (2.5), then there exists a unique nonsingular matrix $M \in C^{n \times n}$ such that

$$
\Phi(t+T)=\Phi(t) M \quad(t \in \mathbb{R}) .
$$

By equality (2.6) there corresponds to the fundamental matrix $\Phi(t)$ and the constant matrix $M$ which we call the monodromy matrix of (2.5) (corresponding to the fundamental matrix of $\Phi(t))$. All monodromy matrices of (2.5) are similar and have the same eigenvalues. The eigenvalues $\mu_{1}, \ldots, \mu_{n}$ of the monodromy matrices are called the Floquet multipliers of (2.5).

Lemma 2.4 (Floquet theory [14]). Let conditions $\left(H_{1}\right)-\left(H_{3}\right)$ hold. Then the linear T-periodic impulsive equation (2.5) is

(1) stale if and only if all multipliers $\mu_{j}(j=1, \ldots, n)$ of (2.5) satisfy the inequality $\left|\mu_{j}\right| \leq 1$, and moreover, to those $\mu_{j}$ for which $\left|\mu_{j}\right|=1$, there correspond simple elementary divisors;

(2) asymptotically stable if and only if all multipliers $\mu_{j}(j=1, \ldots, n)$ of $(2.5)$ satisfy the inequality $\left|\mu_{j}\right|<1$;

(3) unstable if $\left|\mu_{j}\right|>1$ for some $j=1, \ldots, n$.

\section{Mathematical Analysis}

In this section, we have focused on two main subjects, one is about the extinction of the prey and the other is about the coexistence of the prey and the predator. For the extinction, we have found out a condition that the population of the prey goes to zero as time goes by via the study of the stability of a prey-free periodic solution. For example, if the prey is regarded as a pest, it is important to figure out when the population of the prey dies out. For the reason, it is necessary to consider the stability of the prey-free periodic solution. On the other hand, for the coexistence, we have investigated that the populations of the prey and the predator become positive and finite under certain conditions. 


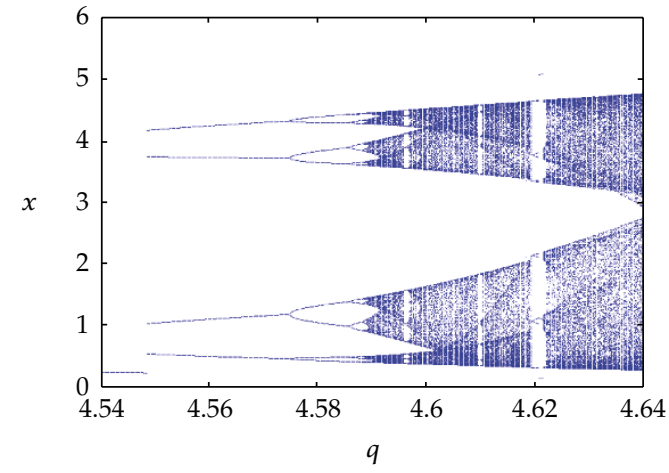

(a)

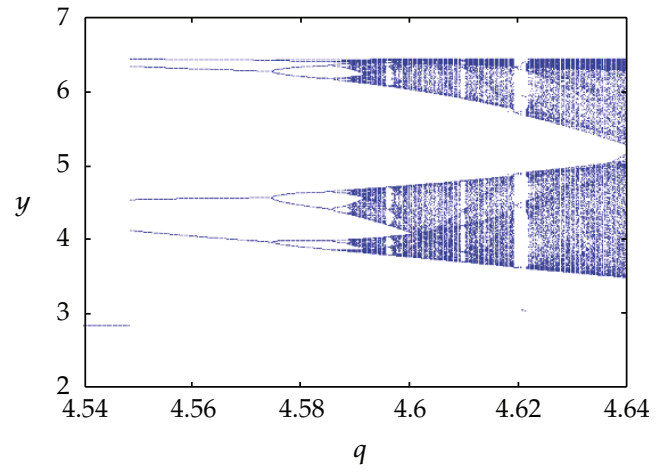

(b)

Figure 4: Bifurcation diagrams of (1.3) for $q$ ranging from $4.54<q<4.64$. (a) $x$ is plotted. (b) $y$ is plotted.

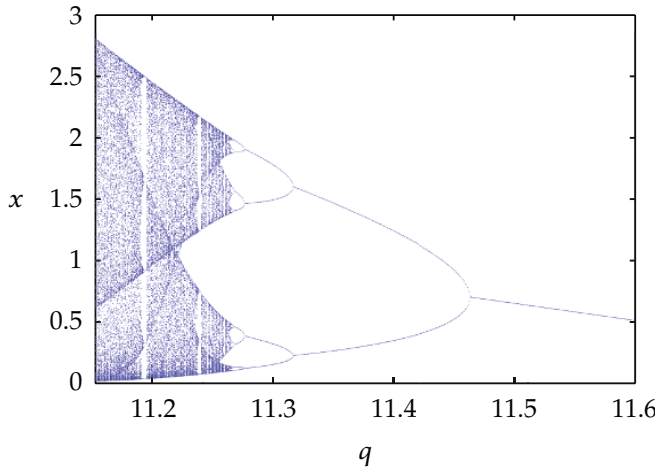

(a)

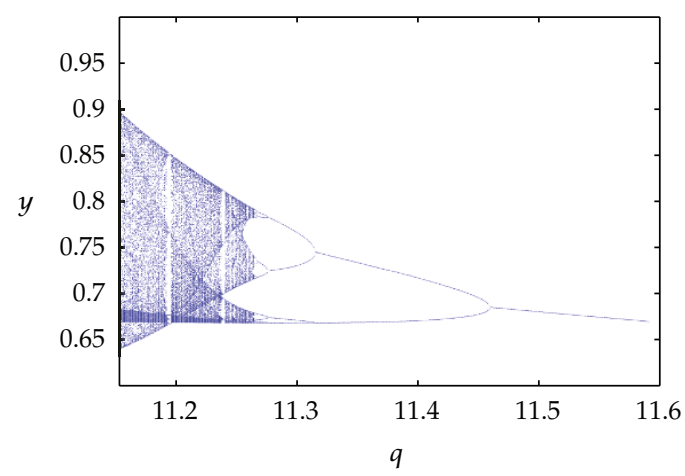

(b)

Figure 5: Bifurcation diagrams of (1.3) for $q$ ranging from $11.153<q<11.6$. (a) $x$ is plotted. (b) $y$ is plotted.

\subsection{Stability for a Prey-Free Periodic Solution}

First of all, in order to study the extinction of the prey, the existence of a prey-free solution to the system (1.3) should be guaranteed. For the reason, we give some basic properties of the following impulsive differential equation which comes from the system (1.3) by setting $x(t)=0$

$$
\begin{gathered}
y^{\prime}(t)=-d y(t), \quad t \neq n T, t \neq(n+\tau-1) T, \\
y\left(t^{+}\right)=\left(1-p_{2}\right) y(t), \quad t=(n+\tau-1) T, \\
y\left(t^{+}\right)=y(t)+q, \quad t=n T, \\
y\left(0^{+}\right)=y_{0} .
\end{gathered}
$$


The system (3.1) is a periodically forced linear system; it is easy to obtain from elementary calculations that

$$
y^{*}(t)= \begin{cases}\frac{q \exp (-d(t-(n-1) T))}{1-\left(1-p_{2}\right) \exp (-d T)}, & (n-1) T<t \leq(n+\tau-1) T, \\ \frac{q\left(1-p_{2}\right) \exp (-d(t-(n-1) T))}{1-\left(1-p_{2}\right) \exp (-d T)}, & (n+\tau-1) T<t \leq n T,\end{cases}
$$

$y^{*}\left(0^{+}\right)=y^{*}\left(n T^{+}\right)=\left(q / 1-\left(1-p_{2}\right) \exp (-d T)\right), y^{*}\left(\tau T^{+}\right)=\left(q\left(1-p_{2}\right) \exp (-d \tau T) / 1-(1-\right.$ $\left.\left.p_{2}\right) \exp (-d T)\right)$ is a positive periodic solution of (3.1). Moreover, we can figure out that

$$
y(t)=\left\{\begin{array}{c}
\left(1-p_{2}\right)^{n-1}\left(y\left(0^{+}\right)-\frac{q\left(1-p_{2}\right) e^{-T}}{1-\left(1-p_{2}\right) \exp (-d T)}\right) \exp (-d t)+y^{*}(t), \\
(n-1) T<t \leq(n+\tau-1) T, \\
\left(1-p_{2}\right)^{n}\left(y\left(0^{+}\right)-\frac{q\left(1-p_{2}\right) \mathrm{e}^{-T}}{1-\left(1-p_{2}\right) \exp (-d T)}\right) \exp (-d t)+y^{*}(t), \\
(n+\tau-1) T<t \leq n T,
\end{array}\right.
$$

is the solution of (3.1). From (3.2) and (3.3), the following results can be easily obtained without the proof.

Lemma 3.1. For every solution $y(t)$ and every positive periodic solution $y^{*}(t)$ of the system (3.1), it follows that $y(t)$ tend to $y^{*}(t)$ as $t \rightarrow \infty$. Thus, the complete expression for the prey-free periodic solution of the system (1.3) is obtained $\left(0, y^{*}(t)\right)$.

Now, in the next theorem, the stability of the periodic solution $\left(0, y^{*}(t)\right)$ is investigated.

Theorem 3.2. Let $(x(t), y(t))$ be any solution of the system (1.3). Then the prey-free periodic solution $\left(0, y^{*}(t)\right)$ is locally asymptotically stable if

$$
a T-\frac{c q\left(1+\left(p_{2}-1\right) \exp (-d T)-p_{2} \exp (-d \tau T)\right)}{d\left(1-\left(1-p_{2}\right) \exp (-d T)\right)}<\ln \frac{1}{1-p_{1}}
$$

Proof. The local stability of the periodic solution $\left(0, y^{*}(t)\right)$ of the system (1.3) may be determined by considering the behavior of small amplitude perturbations of the solution. Define $x(t)=u(t), y(t)=y^{*}(t)+v(t)$. Then they may be written as

$$
\left(\begin{array}{l}
u(t) \\
v(t)
\end{array}\right)=\Phi(t)\left(\begin{array}{l}
u(0) \\
v(0)
\end{array}\right)
$$


where $\Phi(t)$ satisfies

$$
\frac{d \Phi}{d t}=\left(\begin{array}{cc}
a-c y^{*}(t) & 0 \\
e y^{*}(t) & -d
\end{array}\right) \Phi(t)
$$

and $\Phi(0)=I$, the identity matrix. So the fundamental solution matrix is

$$
\Phi(t)=\left(\begin{array}{cc}
\exp \left(\int_{0}^{t} a-c y^{*}(s) d s\right) & 0 \\
\exp \left(e \int_{0}^{t} y^{*}(s) d s\right) & \exp (-d t)
\end{array}\right) .
$$

The resetting impulsive condition of the system (1.3) becomes

$$
\begin{gathered}
\left(\begin{array}{c}
u\left((n+\tau-1) T^{+}\right) \\
v\left((n+\tau-1) T^{+}\right)
\end{array}\right)=\left(\begin{array}{cc}
1-p_{1} & 0 \\
0 & 1-p_{2}
\end{array}\right)\left(\begin{array}{l}
u((n+\tau-1) T) \\
v((n+\tau-1) T)
\end{array}\right) \\
\left(\begin{array}{l}
u\left(n T^{+}\right) \\
v\left(n T^{+}\right)
\end{array}\right)=\left(\begin{array}{ll}
1 & 0 \\
0 & 1
\end{array}\right)\left(\begin{array}{l}
u(n T) \\
v(n T)
\end{array}\right) .
\end{gathered}
$$

Note that all eigenvalues of

$$
S=\left(\begin{array}{cc}
1-p_{1} & 0 \\
0 & 1-p_{2}
\end{array}\right)\left(\begin{array}{ll}
1 & 0 \\
0 & 1
\end{array}\right) \Phi(T)
$$

are $\mu_{1}=\left(1-p_{2}\right) \exp (-d T)<1$ and $\mu_{2}=\left(1-p_{1}\right) \exp \left(\int_{0}^{T} a-c y^{*}(t) d t\right)$. Since

$$
\int_{0}^{T} y^{*}(t) d t=\frac{q\left(1+\left(p_{2}-1\right) \exp (-d T)-p_{2} \exp (-d \tau T)\right)}{d\left(1-\left(1-p_{2}\right) \exp (-d T)\right)}
$$

the condition $\left|\mu_{2}\right|<1$ is equivalent to the equation

$$
a T-\frac{c q\left(1+\left(p_{2}-1\right) \exp (-d T)-p_{2} \exp (-d \tau T)\right)}{d\left(1-\left(1-p_{2}\right) \exp (-d T)\right)}<\ln \frac{1}{1-p_{1}} .
$$

According to Lemma $2.4,\left(0, y^{*}(t)\right)$ is locally stable.

Remark 3.3. (1) It follows from Theorem 3.2 that the population of the prey could be controlled by using chemical or biological control parameters, $p_{1}, p_{2}, q$ if the other parameters are fixed. (2) Figure 2 illustrates this phenomenon. 


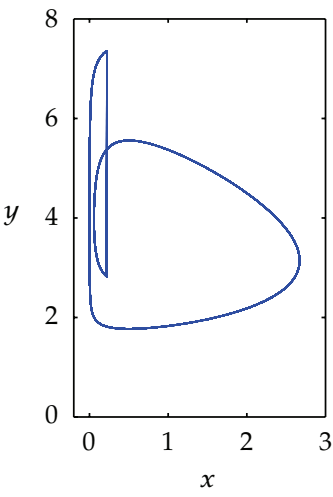

(a)

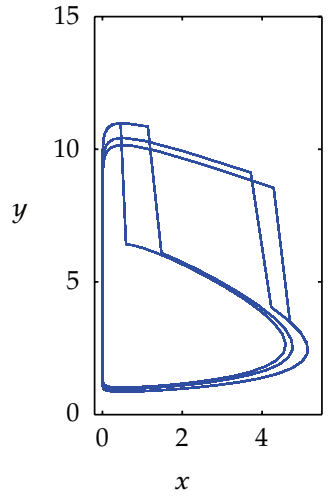

(b)

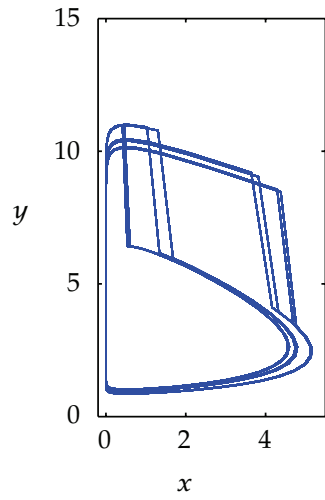

(c)

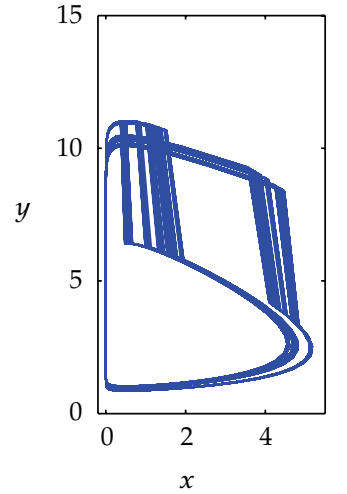

(d)

Figure 6: Phase portrait of (1.3). (a) $q=4.54$. (b) $q=4.57$. (c) $q=4.58$. (d) $q=4.595$.

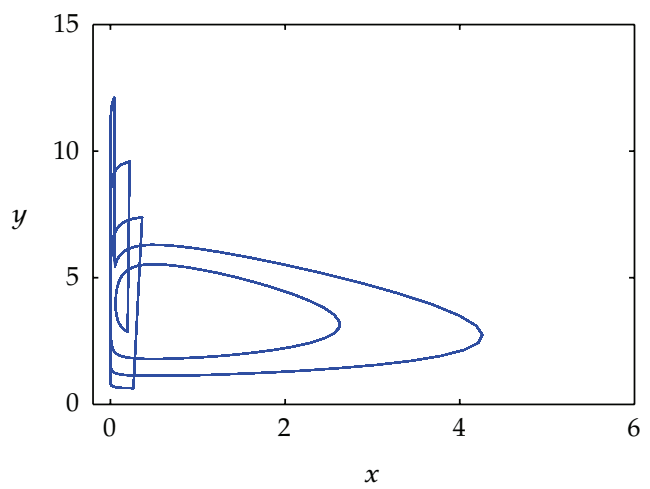

(a)

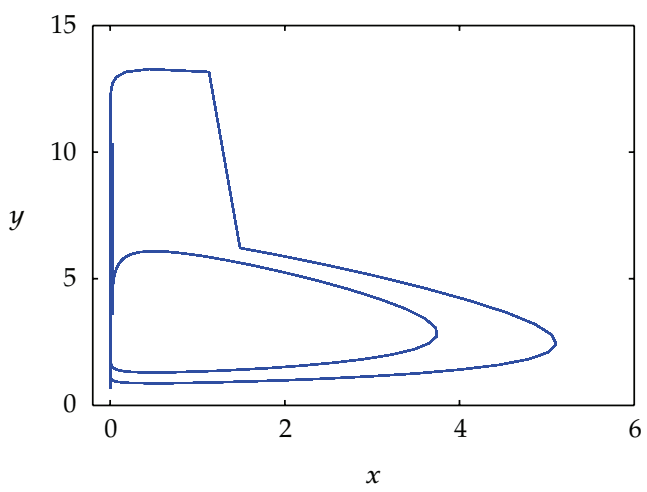

(b)

Figure 7: Coexistence of solutions when $p=6.755$. (a) Solution with initial values $(1,1)$. (b) Solution with initial values $(1.3,2.9)$.

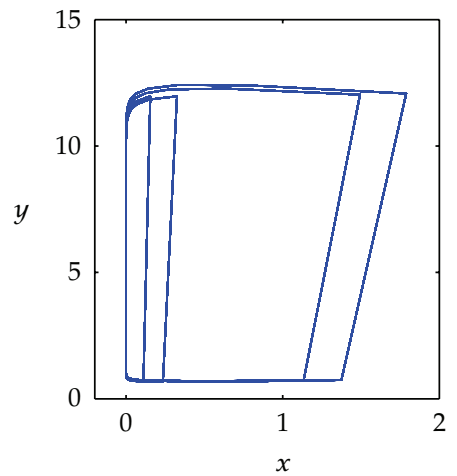

(a)

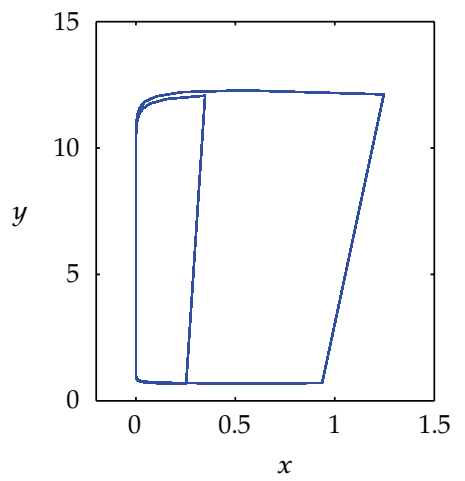

(b)

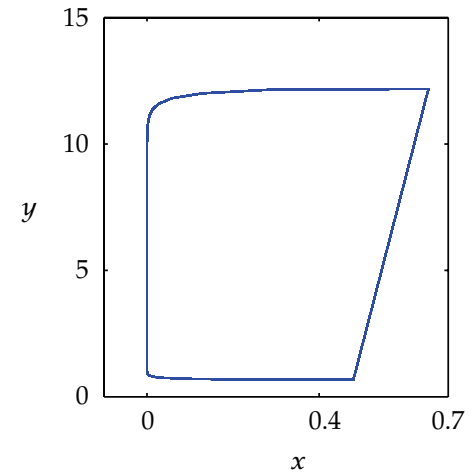

(c)

Figure 8: Period-halving bifurcation from 4T-periodic solutions to cycles of (1.3). (a) Phase portrait of a $4 T$-period solution for $q=11.3$. (b) Phase portrait of a $2 T$-period solution for $q=11.4$. (c) Phase portrait of a $T$-period solution for $q=11.5$. 


\subsection{Permanence}

It might be difficult to find out a necessary condition for the stability of the prey-free periodic solution $\left(0, y^{*}(t)\right)$. Due to this fact, it is natural to have a question what a condition that makes all species coexist is. Before answering the question, first of all, we introduce a definition which keeps the concept of coexistence of the prey and the predator.

Definition 3.4. The system (1.3) is permanent if there exists $M \geq m>0$ such that, for any solution $(x(t), y(t))$ of the system (1.3) with $x_{0}>0$,

$$
m \leq \lim _{t \rightarrow \infty} \inf x(t) \leq \lim _{t \rightarrow \infty} \sup x(t) \leq M, \quad m \leq \lim _{t \rightarrow \infty} \inf y(t) \leq \lim _{t \rightarrow \infty} \sup y(t) \leq M .
$$

From a biological point of view, the populations of the prey and the predator in the system (1.3) cannot increase up to infinity due to restriction of resources. To show this phenomenon for the system (1.3) mathematically, we prove that all solutions to the system (1.3) are uniformly ultimately bounded in the next proposition.

Proposition 3.5. There is an $M>0$ such that $x(t), y(t) \leq M$ for all $t$ large enough, where $(x(t), y(t))$ is a solution of the system (1.3).

Proof. Let $\mathbf{x}(t)=(x(t), y(t))$ be a solution of the system (1.3) and let $V(t, \mathbf{x})=e x(t)+c y(t)$. Then $V \in V_{0}$, if $t \neq(n+\tau-1) T$ and $t \neq(n+\tau) T$

$$
D^{+} V+\beta V=-\frac{e a}{K} x(t)^{2}+e(a+\beta) x(t)+c(\beta-d) y(t) .
$$

When $t=(n+\tau-1) T, V\left((n+\tau-1) T^{+}\right) \leq V((n+\tau-1) T)$ and when $t=n T, V\left(n T^{+}\right) \leq V(n T)+q$. Clearly, the right hand of (3.13), is bounded when $0<\beta<d$. So we can choose $0<\beta_{0}<d$ and $M_{0}>0$ such that

$$
\begin{gathered}
D^{+} V \leq-\beta_{0} V+M_{0}, \quad t \neq(n+\tau-1) T, t \neq n T, \\
V\left(t^{+}\right) \leq V(t), \quad t=(n+\tau-1) T, \\
V\left(t^{+}\right) \leq V(t)+q, \quad t=n T .
\end{gathered}
$$

By Lemma 2.2, we can obtain that

$$
V(t) \leq V\left(0^{+}\right) \exp \left(-\beta_{0} t\right)+\frac{M_{0}}{\beta_{0}}\left(1-\exp \left(-\beta_{0} t\right)\right)+\frac{q\left(\exp \left(-\left(\beta_{0}+1\right) T\right)-\exp \left(-\beta_{0}(t-(n-1) T)\right)\right)}{1-\exp \left(-\beta_{0} T\right)}
$$

for $t \in((n-1) T, n T]$. Therefore, $V(t)$ is bounded by a constant for sufficiently large $t$. Hence there is an $M>0$ such that $x(t) \leq M, y(t) \leq M$ for a solution $(x(t), y(t))$ with all $t$ large enough.

Thanks to Proposition 3.5, we have only to prove the existence of a positive lower bound for the populations of the prey and the predator to justify the system is permanent. 
Theorem 3.6. The system (1.3) is permanent if

$$
a T-\frac{c q\left(1+\left(p_{2}-1\right) \exp (-d T)-p_{2} \exp (-d \tau T)\right)}{d\left(1-\left(1-p_{2}\right) \exp (-d T)\right)}>\ln \frac{1}{1-p_{1}}
$$

Proof. Suppose $(x(t), y(x))$ is any solution of the system (1.3) with $\mathbf{x}_{0}>0$. From Proposition 3.5, we may assume that $x(t) \leq M, y(t) \leq M, t \geq 0$ and $M>a / c$. Let $m_{2}=q\left(1-p_{2}\right) \exp (-d T) /\left(1-\left(1-p_{2}\right) \exp (-d T)-\epsilon_{2}\right), \epsilon_{2}>0$. So, it is easily induced from Lemma 3.1 that $y(t) \geq m_{2}$ for all $t$ large enough. Now we shall find an $m_{1}>0$ such that $x(t) \geq m_{1}$ for all $t$ large enough. We will do this in the following two steps.

Step 1. Since

$$
a T-\frac{c q\left(1+\left(p_{2}-1\right) \exp (-d T)-p_{2} \exp (-d \tau T)\right)}{d\left(1-\left(1-p_{2}\right) \exp (-d T)\right)}>\ln \frac{1}{1-p_{1}}
$$

we can choose $m_{3}>0, \epsilon_{1}>0$ small enough such that $\delta=\left(e m_{3} m_{2} / 1+b m_{3}^{2}\right)<d$ and $R=(1-$ $\left.p_{1}\right) \exp \left(-c q\left(1+\left(p_{2}-1\right) \exp (b(-d+\delta) T)-p_{2} \exp ((-d+\delta) \tau T)\right) /(d-\delta)\left(1-\left(1-p_{2}\right) \exp ((-d+\delta) T)\right)+\right.$ $\left.a T-(a / K) T m_{3}-c \epsilon_{1} T\right)>1$. Suppose that $x(t)<m_{3}$ for all $t$. Then we get $y^{\prime}(t) \leq(-d+\delta) y(t)$ from above assumptions. By Lemma 2.2, we have $y(t) \leq u(t)$ and $u(t) \rightarrow u^{*}(t), t \rightarrow \infty$, where $u(t)$ is the solution of

$$
\begin{gathered}
u^{\prime}(t)=(-d+\delta) u(t), \quad t \neq(n+\tau-1) T, t \neq n T, \\
u\left(t^{+}\right)=\left(1-p_{2}\right) u(t), \quad t=(n+\tau-1) T, \\
u\left(t^{+}\right)=u(t)+q, \quad t=n T, \\
u\left(0^{+}\right)=y\left(0^{+}\right),
\end{gathered} \quad \begin{array}{cc}
\frac{q \exp ((-d+\delta)(t-(n-1) T))}{1-\left(1-p_{2}\right) \exp ((-d+\delta) T)}, & (n-1) T<t \leq(n+\tau-1) T, \\
u^{*}(t)=\left\{\begin{array}{c}
q\left(1-p_{2}\right) \exp ((-d+\delta)(t-(n-1) T)) \\
\frac{1-\left(1-p_{2}\right) \exp ((-d+\delta) T)}{1-(n+\tau-1) T<t \leq n T .}
\end{array}\right.
\end{array}
$$

Then there exists $T_{1}>0$ such that $y(t) \leq u(t) \leq u^{*}(t)+\epsilon_{1}$ for $t \geq T_{1}$. So we obtain that

$$
\begin{aligned}
x^{\prime}(t) & =x(t)\left(a-\frac{a}{K} x(t)\right)-\frac{c x(t) y(t)}{1+b x^{2}(t)} \\
& \geq x(t)\left(a-\frac{a}{K} m_{3}-c y(t)\right) \\
& \geq x(t)\left(a-\frac{a}{K} m_{3}-c\left(u^{*}(t)+\epsilon_{1}\right)\right), \quad t \neq(n+\tau-1) T, \\
& x\left(t^{+}\right)=\left(1-p_{1}\right) x(t), \quad t=(n+\tau-1) T,
\end{aligned}
$$


for $t \geq T_{1}$. Let $N_{1} \in \mathbb{N}$ and $\left(N_{1}+\tau-1\right) T \geq T_{1}$. Integrating (3.20) on $((n+\tau-1) T,(n+\tau) T], n \geq N_{1}$, we have $x((n+\tau) T) \geq x((n+\tau-1) T)\left(1-p_{1}\right) \exp \left(\int_{(n+\tau-1) T}^{(n+\tau) T} a-(a / K) m_{3}-c\left(u^{*}(t)+\epsilon_{1}\right) d t\right)=$ $x((n+\tau-1) T) R$. Then we have $x\left(\left(N_{1}+\tau+n\right) T\right) \geq x\left(\left(N_{1}+\tau\right) T\right) R^{n} \rightarrow \infty$ as $n \rightarrow \infty$ which is a contradiction. Hence there exists a $t_{1}>0$ such that $x\left(t_{1}\right) \geq m_{3}$.

Step 2. If $x(t) \geq m_{3}$ for all $t \geq t_{1}$, then we are done. If not, we may let $t^{*}=\inf _{t>t_{1}}\left\{x(t)<m_{3}\right\}$. Then $x(t) \geq m_{3}$ for $t \in\left[t_{1}, t^{*}\right]$ and, by the continuity of $x(t)$, we have $x\left(t^{*}\right)=m_{3}$. In this step, we have only to consider two possible cases.

Case 1. $t^{*}=\left(n_{1}+\tau-1\right) T$ for some $n_{1} \in \mathbb{N}$. Then $\left(1-p_{1}\right) m_{3} \leq x\left(t^{*+}\right)=\left(1-p_{1}\right) x\left(t^{*}\right)<m_{3}$. Select $n_{2}, n_{3} \in \mathbb{N}$ such that $\left(n_{2}-1\right) T>\ln \left(\epsilon_{1} / M+q\right) /(-d+\delta)$ and $\left(1-p_{1}\right)^{n_{2}} R^{n_{3}} \exp \left(n_{2} \sigma T\right)>$ $\left(1-p_{1}\right)^{n_{2}} R^{n_{3}} \exp \left(\left(n_{2}+1\right) \sigma T\right)>1$, where $\sigma=a-(a / K) m_{3}-c M<0$. Let $T^{\prime}=n_{2} T+n_{3} T$. In this case we will show that there exists $t_{2} \in\left(t^{*}, t^{*}+T^{\prime}\right]$ such that $x\left(t_{2}\right) \geq m_{3}$. Otherwise, by (3.18) with $u\left(n_{1} T^{+}\right)=y\left(n_{1} T^{+}\right)$, we have

$$
u(t)=\left\{\begin{array}{c}
\left(1-p_{2}\right)^{n-\left(n_{1}+1\right)}\left(u\left(n_{1} T^{+}\right)-\frac{q\left(1-p_{2}\right) \exp (-T)}{1-\left(1-p_{2}\right) \exp ((-d+\delta) T)}\right) \\
\exp \left((-d+\delta)\left(t-n_{1} T\right)\right)+u^{*}(t), \quad(n-1) T<t \leq(n+\tau-1) T, \\
\left(1-p_{2}\right)^{\left(n-n_{1}\right)}\left(u\left(n_{1} T^{+}\right)-\frac{q\left(1-p_{2}\right) \exp (-T)}{1-\left(1-p_{2}\right) \exp ((-d+\delta) T)}\right) \\
\exp \left((-d+\delta)\left(t-n_{1} T\right)\right)+u^{*}(t), \quad(n+\tau-1) T<t \leq n T,
\end{array}\right.
$$

and $n_{1}+1 \leq n \leq n_{1}+1+n_{2}+n_{3}$. So we get $\left|u(t)-u^{*}(t)\right| \leq(M+q) \exp \left((-d+\delta)\left(t-n_{1} T\right)\right)<\epsilon_{1}$ and $y(t) \leq u(t) \leq u^{*}(t)+\epsilon_{1}$ for $n_{1} T+\left(n_{2}-1\right) T \leq t \leq t^{*}+T^{\prime}$. Also we get to know that

$$
\begin{gathered}
x^{\prime}(t) \geq x(t)\left(a-\frac{a}{K} m_{3}-c\left(u^{*}+\epsilon_{1}\right)\right), \quad t \neq(n+\tau-1) T, \\
x\left(t^{+}\right)=\left(1-p_{1}\right)(t), \quad t=(n+\tau-1) T
\end{gathered}
$$

for $t \in\left[t^{*}+n_{2} T, t^{*}+T^{\prime}\right]$. As in Step 1, we have

$$
x\left(t^{*}+T^{\prime}\right) \geq x\left(t^{*}+n_{2} T\right) R^{n_{3}} .
$$

Since $y(t) \leq M$, we have

$$
\begin{gathered}
x^{\prime}(t) \geq x(t)\left(a-\frac{a}{K} m_{3}-c M\right)=\sigma x(t), \quad t \neq n T, \\
x\left(t^{+}\right)=\left(1-p_{1}\right) x(t), \quad t=n T,
\end{gathered}
$$

for $t \in\left[t^{*}, t^{*}+n_{2} T\right]$. Integrating (3.24) on $\left[t^{*}, t^{*}+n_{2} T\right]$ we have

$$
x\left(\left(t^{*}+n_{2} T\right)\right) \geq m_{3} \exp \left(\sigma n_{2} T\right) \geq m_{3}\left(1-p_{1}\right)^{n_{2}} \exp \left(\sigma n_{2} T\right)>m_{3} .
$$


Thus $x\left(t^{*}+T^{\prime}\right) \geq m_{3}\left(1-p_{1}\right)^{n_{2}} \exp \left(\sigma n_{2} T\right) R^{n_{3}}$ which is a contradiction. Now, let $\bar{t}=\inf _{t>t^{*}}\{x(t) \geq$ $\left.m_{3}\right\}$. Then $x(t) \leq m_{3}$ for $t^{*} \leq t<\bar{t}$ and $x(\bar{t})=m_{3}$. So, we have, for $t \in\left[t^{*}, \bar{t}\right), x(t) \geq m_{3}(1-$ $\left.\mathrm{p}_{1}\right)^{n_{2}+n_{3}} \exp \left(\sigma\left(n_{2}+n_{3}\right) T\right) \equiv m_{1}^{\prime}$.

Case 2. $t^{*} \neq(n+\tau-1) T, n \in \mathbb{N}$. Suppose that $t^{*} \in\left(\left(n_{1}^{\prime}+\tau-1\right) T,\left(n_{1}^{\prime}+\tau\right) T\right), n_{1}^{\prime} \in \mathbb{N}$. There are two possible cases for $t \in\left(t^{*},\left(n_{1}^{\prime}+\tau\right) T\right)$.If $x(t) \leq m_{3}$ for all $t \in\left(t^{*},\left(n^{\prime} 1+\tau\right) T\right)$, similar to (Case 1), we can prove there must be a $t_{2}^{\prime} \in\left[\left(n_{1}^{\prime}+\tau\right) T,\left(n_{1}^{\prime}+\tau\right) T+T^{\prime}\right]$ such that $x\left(t_{2}^{\prime}\right) \geq m_{3}$. Here we omit it. Let $\widehat{t}=\inf _{t>t^{*}}\left\{x(t) \geq m_{3}\right\}$. Then $x(t) \leq m_{3}$ for $t \in\left(t^{*}, \widehat{t}\right)$ and $x(\widehat{t})=m_{3}$. For $t \in\left(t^{*}, \widehat{t}\right)$, we have $x(t) \geq m_{3}\left(1-p_{1}\right)^{n_{2}+n_{3}} \exp \left(\sigma_{1}\left(n_{2}+n_{3}+1\right) T\right)=m_{1}$. So, $m_{1}<m_{1^{\prime}}$ and $x(t) \geq m_{1}$ for $t \in\left(t^{*}, \widehat{t}\right)$. If there exists a $t \in\left(t^{*},\left(n_{1}^{\prime}+\tau\right) T\right)$ such that $x_{1}(t) \geq m_{3}$. Let $\breve{t}=\inf _{t>t^{*}}\left\{x(t) \geq m_{3}\right\}$. Then $x(t) \leq m_{3}$ for $t \in\left(t^{*}, \check{t}\right)$ and $x(\breve{t})=m_{3}$. For $t \in\left(t^{*}, \breve{t}\right)$, we have $x(t) \geq x\left(t^{*}\right) \exp \left(\sigma\left(t-t^{*}\right)\right) \geq m_{3} \exp (\sigma T)>m_{1}$.

Thus in both case the similar argument can be continued since $x(t) \geq m_{3}$ for some $t>t_{1}$. This completes the proof.

Remark 3.7. (1) Figures 1 and 7 are numerical evidences which satisfy the conditions of Theorem 3.6. (2) Theorem 2.1 and 2.3 in [20] can be obtained as corollaries of Theorem 3.2 and 3.6, respectively, by taking $p_{1}=p_{2}=0$ in the system (1.3).

\section{Numerical Analysis on Impulsive Perturbations}

It is well known that the continuous system (1.3) cannot be solved explicitly. Thus we should study the system (1.3) by using numerical method and research the long-term behavior of the solutions to get more information about the dynamic behaviors of the system (1.3). We thus numerically investigate the influence of impulsive perturbation. For this, we fix the parameters except the control parameters $p_{1}, p_{2}$ and $q$ as follows:

$$
\begin{aligned}
a=4, \quad K=10, \quad b & =0.01, \quad c=1, \quad d=0.2, \quad e=0.4, \\
p_{1}=0.2, \quad p_{2} & =0.0001, \quad \tau=0.2, \quad T=15 .
\end{aligned}
$$

It is from [5] that the system (1.3) with $p_{1}=p_{2}=0$ and $q=0$ has an unique limit cycle. Moreover, Figure 1 shows that the phase portrait of the system (1.3) with $p_{1}=0.2$, $p_{2}=0.0001$, and $q=0$ has a limit cycle too. From Theorem 3.2, we know that the prey-free periodic solution $\left(0, y^{*}(t)\right)$ is locally asymptotically stable provided that $q>q_{\max }=11.9561$. A typical prey-free periodic solution $\left(o, y^{*}(t)\right)$ of the system (1.3) is shown in Figure 2, where we observe how the variable $y(t)$ oscillates in a stable cycle while the prey $x(t)$ rapidly decreases to zero. On the other hand, if the amount $q$ of releasing species is smaller than $q_{\max }$, then the prey and the predator can coexist on a stable positive periodic solution (see Figure 1) and the system (1.3) can be permanent, which follows from Theorem 3.6.

Now we investigate the effect of impulsive perturbations. In Figure 3, we displayed bifurcation diagrams for the prey and predator populations as $q$ increasing from 0 to 13 with an initial value $x_{0}=(1,1)$. The resulting bifurcation diagram clearly show that the system (1.3) has rich dynamics including cycles, periodic doubling bifurcation, chaotic bands, periodic window, and period-halving bifurcation. Figures 4 and 5 are the magnified parts of Figure 3, and the windows of periodic behaviors are more visible.

As is evident from Figure 3, the solutions of the system (1.3) are $T$-periodic when $q<$ 2.372 and $3.8025<q<4.5338$ and $2 T$-periodic when $2.372<q<3.8025$. Generally, periodic 
doubling leads to chaos. We can take a local view of this phenomenon in Figure 4. But Figures 4 and 6 show the route to chaos through the cascade of period four. This phenomenon is caused by sudden changes when $q \approx 4.5847$. We can also find such phenomena when $q \approx$ $4.6207,5.3834,6.755,9,709$, and so on. One of interesting things is that they can lead to nonunique attractors. In fact, Figure 7 exhibits the existence of multiattractors when $q \approx 6.755$. These results show that just one parameter could give rise to multiple attractors. Narrow periodic windows and wide periodic windows are intermittently scattered (see Figure 3 ). At the end of the chaotic region, there is a cascade of period-halving bifurcation from chaos to one cycle. (see Figures 5 and 8). Periodic halving is the flip bifurcation in the opposite direction.

\section{Discussion}

In this paper, we have studied the effects of control strategies on a predator-prey system with Monod-Haldane type functional response. Conditions for the system to be extinct are given by using the Floquet theory of impulsive differential equation and small amplitude perturbation skills. Also, it is proved that the system the system (1.3) is permanent via the comparison theorem. Moreover, numerical examples on impulsive perturbations have been illustrated to substantiate our mathematical results and to show that the system we have considered in this paper gives birth to various kinds of dynamical behaviors.

Actually, in the real world, there are a number of environmental factors we should consider to describe the world more realistically. Among them, seasonal effect on the prey is one of the most important factors in the ecological systems. There are many ways to apply such phenomena in an ecological system $[22,23]$. In this context, we think about the intrinsic growth rate $a$ in the system (1.3) as periodically varying function of time due to seasonal variation, which is superimposed as follows:

$$
a_{0}=a(1+\epsilon \sin (\omega t))
$$

where the parameter $\epsilon$ represents the degree of seasonality, $\lambda=a \epsilon$ is the magnitude of the perturbation in $a_{0}$ and $\omega$ is the angular frequency of the fluctuation caused by seasonality. Now, the system (1.3) can be changed as follows:

$$
\begin{aligned}
& x^{\prime}(t)=a x(t)\left(1-\frac{x(t)}{K}\right)-\frac{c x(t) y(t)}{1+b x^{2}(t)}+\lambda x(t) \sin (\omega t), \quad t \neq n T, \quad t \neq(n+\tau-1) T, \\
& \begin{aligned}
& y^{\prime}(t)=-d y(t)+\frac{e x(t) y(t)}{1+b x^{2}(t)} \\
& x\left(t^{+}\right)=\left(1-p_{1}\right) x(t), \quad t=(n+\tau-1) T, \\
& y\left(t^{+}\right)=\left(1-p_{2}\right) y(t), \\
& x\left(t^{+}\right)=x(t), \\
& y\left(t^{+}\right)=y(t)+q, \quad t=n T, \\
&\left(x\left(0^{+}\right), y\left(0^{+}\right)\right)=\left(x_{0}, y_{0}\right)=\mathbf{x}_{0} .
\end{aligned}
\end{aligned}
$$

And then we get the following results via similar methods used in the previous sections. 
Theorem 5.1. Let $(x(t), y(t))$ be any solution of the system (5.2). Then the prey-free periodic solution $\left(0, y^{*}(t)\right)$ is locally asymptotically stable if

$$
a T+\frac{\lambda}{\omega}(1-\cos (\omega t))-\frac{c q\left(1+\left(p_{2}-1\right) \exp (-d T)-p_{2} \exp (-d \tau T)\right)}{d\left(1-\left(1-p_{2}\right) \exp (-d T)\right)}<\ln \frac{1}{1-p_{1}} .
$$

Proposition 5.2. There is an $M>0$ such that $x(t), y(t) \leq M$ for all $t$ large enough, where $(x(t), y(t))$ is a solution of the system (5.2).

Theorem 5.3. The system (5.2) is permanent if

$$
(a-\lambda) T-\frac{c q\left(1+\left(p_{2}-1\right) \exp (-d T)-p_{2} \exp (-d \tau T)\right)}{d\left(1-\left(1-p_{2}\right) \exp (-d T)\right)}>\ln \frac{1}{1-p_{1}} .
$$

Thus, the seasonal effect on the prey may have also deeply influences on dynamics of the system (1.3).

\section{Acknowledgments}

The first author is supported by Catholic University of Daegu Research Grant. The second author was supported by Basic Science Research Program through the National Research Foundation of Korea (NRF) funded by the Ministry of Education, Science and Technology (2010-0004426).

\section{References}

[1] Z. Lu, X. Chi, and L. Chen, "Impulsive control strategies in biological control of pesticide," Theoretical Population Biology, vol. 64, no. 1, pp. 39-47, 2003.

[2] S. Tang, Y. Xiao, L. Chen, and R. A. Cheke, "Integrated pest management models and their dynamical behaviour," Bulletin of Mathematical Biology, vol. 67, no. 1, pp. 115-135, 2005.

[3] C. S. Holling, "The functional response of predator to prey density and its role in mimicry and population regulations," Memoirs of the Entomological Society of Canada, vol. 45, pp. 1-60, 1965.

[4] S. B. Hsu and T. W. Huang, "Global stability for a class of predator-prey systems," SIAM Journal on Applied Mathematics, vol. 55, no. 3, pp. 763-783, 1995.

[5] S. Ruan and D. Xiao, "Global analysis in a predator-prey system with nonmonotonic functional response," SIAM Journal on Applied Mathematics, vol. 61, no. 4, pp. 1445-1472, 2001.

[6] H. K. Baek, "Qualitative analysis of Beddington-DeAngelis type impulsive predator-prey models," Nonlinear Analysis: Real World Applications, vol. 11, no. 3, pp. 1312-1322, 2010.

[7] J. R. Beddington, "Mutual interference between parasites or predator and its effect on searching efficiency," Journal of Animal Ecology, vol. 44, pp. 331-340, 1975.

[8] M. Fan and Y. Kuang, "Dynamics of a nonautonomous predator-prey system with the BeddingtonDeAngelis functional response," Journal of Mathematical Analysis and Applications, vol. 295, no. 1, pp. 15-39, 2004.

[9] T.-W. Hwang, “Uniqueness of limit cycles of the predator-prey system with Beddington-DeAngelis functional response," Journal of Mathematical Analysis and Applications, vol. 290, no. 1, pp. 113-122, 2004.

[10] J.-W. Feng and S.-H. Chen, "Global asymptotic behavior for the competing predators of the Ivlev types," Mathematica Applicata, vol. 13, no. 4, pp. 85-88, 2000.

[11] V. S. Ivlev, Experimental Ecology of the Feeding of Fishes, Yale University Press, 1961. 
[12] J. Sugie, "Two-parameter bifurcation in a predator-prey system of Ivlev type," Journal of Mathematical Analysis and Applications, vol. 217, no. 2, pp. 349-371, 1998.

[13] W. Sokol and J. A. Howell, "Kineties of phenol oxidation by ashed cell," Biotechnology and Bioengineering, vol. 23, pp. 2039-2049, 1980.

[14] D. D. Bainov and P. S. Simeonov, Impulsive Differential Equations:Periodic Solutions and Applications, vol. 66 of Pitman Monographs and Surveys in Pure and Applied Mathematics, Longman Science \& Technical, Harlo, UK, 1993.

[15] V. Lakshmikantham, D. D. BaInov, and P. S. Simeonov, Theory of Impulsive Differential Equations, vol. 6 of Series in Modern Applied Mathematics, World Scientific, Teaneck, NJ, USA, 1989.

[16] Z. Li, W. Wang, and H. Wang, "The dynamics of a Beddington-type system with impulsive control strategy," Chaos, Solitons \& Fractals, vol. 29, no. 5, pp. 1229-1239, 2006.

[17] B. Liu, Y. Zhang, and L. Chen, "Dynamic complexities in a Lotka-Volterra predator-prey model concerning impulsive control strategy," International Journal of Bifurcation and Chaos, vol. 15, no. 2, pp. 517-531, 2005.

[18] H. Wang and W. Wang, "The dynamical complexity of a Ivlev-type prey-predator system with impulsive effect," Chaos, Solitons \& Fractals, vol. 38, no. 4, pp. 1168-1176, 2008.

[19] W. Wang, H. Wang, and Z. Li, "The dynamic complexity of a three-species Beddington-type food chain with impulsive control strategy," Chaos, Solitons \& Fractals, vol. 32, no. 5, pp. 1772-1785, 2007.

[20] S. Zhang, D. Tan, and L. Chen, "Chaos in periodically forced Holling type IV predator-prey system with impulsive perturbations," Chaos, Solitons E Fractals, vol. 27, no. 4, pp. 980-990, 2006.

[21] S. Zhang, L. Dong, and L. Chen, "The study of predator-prey system with defensive ability of prey and impulsive perturbations on the predator," Chaos, Solitons E Fractals, vol. 23, no. 2, pp. 631-643, 2005.

[22] J. M. Cushing, "Periodic time-dependent predator-prey systems," SIAM Journal on Applied Mathematics, vol. 32, no. 1, pp. 82-95, 1977.

[23] S. Gakkhar and R. K. Naji, "Chaos in seasonally perturbed ratio-dependent prey-predator system," Chaos, Solitons \& Fractals, vol. 15, no. 1, pp. 107-118, 2003. 